\title{
Upper limb pyrophosphate tenosynovitis outside the carpal tunnel
}

\author{
J C GERSTER AND R LAGIER
}

From the Rheumatology and Rehabilitation Centre, University Hospital, CHUV, Lausanne and the Department of Pathology (Osteoarticular Unit), Medical School, Geneva, Switzerland

SUMMARY Three cases of calcium pyrophosphate dihydrate (CPPD) crystal deposits in tendon sheaths outside the carpal tunnel are reported. Crystals were shown by $x$ ray diffraction analysis in one case and by compensated light microscopy in the other two. Surgical excision of the tendon synovial sheath had to be done in two cases (one case with CPPD crystal deposits).

Extra-articular deposition of calcium pyrophosphate dihydrate (CPPD) crystals has been described in rare cases, especially in the ligamentum flavum ${ }^{1-4}$ and in various kinds of tendons: triceps, ${ }^{5}$ flexor digitorum, ${ }^{3}$ and Achilles tendon. ${ }^{6}{ }^{7}$ It has also been reported in bursitis, ${ }^{5}$ in subcutaneous tissue,${ }^{8-10}$ and even in the dura mater. ${ }^{11}$ Presumed similar deposits have been radiologically reported in patients otherwise known for CPPD crystal deposition disease -for example, in transverse ligament of the second cervical vertebra ${ }^{12}$ and in flexor digitorum. ${ }^{13}$ The association of carpal tunnel syndrome with articular chondrocalcinosis has been classically reported ${ }^{14-17}$; deposits of CPPD crystals have been found in the volar carpal ligament as well as in the tendon sheaths. ${ }^{18} 19$

We report the finding of CPPD crystals in the synovial fluid from tendon sheaths outside the carpal tunnel.

\section{Case report}

\section{CAS E 1}

A 72 year old woman had been operated on for a bilateral carpal tunnel syndrome 10 years ago. She had been suffering from pain in the left knee for about five years and from pain in both shoulders for three years. In addition, she had had painful swelling of the ventral part of the fifth left finger for about 10 months. Clinically, the tendon sheath was diffusely swollen and tender and motion of the finger was restricted (Fig. 1). The left knee was

Accepted for publication 9 January 1989.

Correspondence to Dr J C Gerster, Rheumatology and Rehabilitation Centre, University Hospital, CHUV, CH-1011 Lausanne, Switzerland. swollen and tender and the shoulders were limited and tender. Radiographs showed chondrocalcinosis of both wrists, both knees, and a destructive arthropathy of the left shoulder. Degenerative

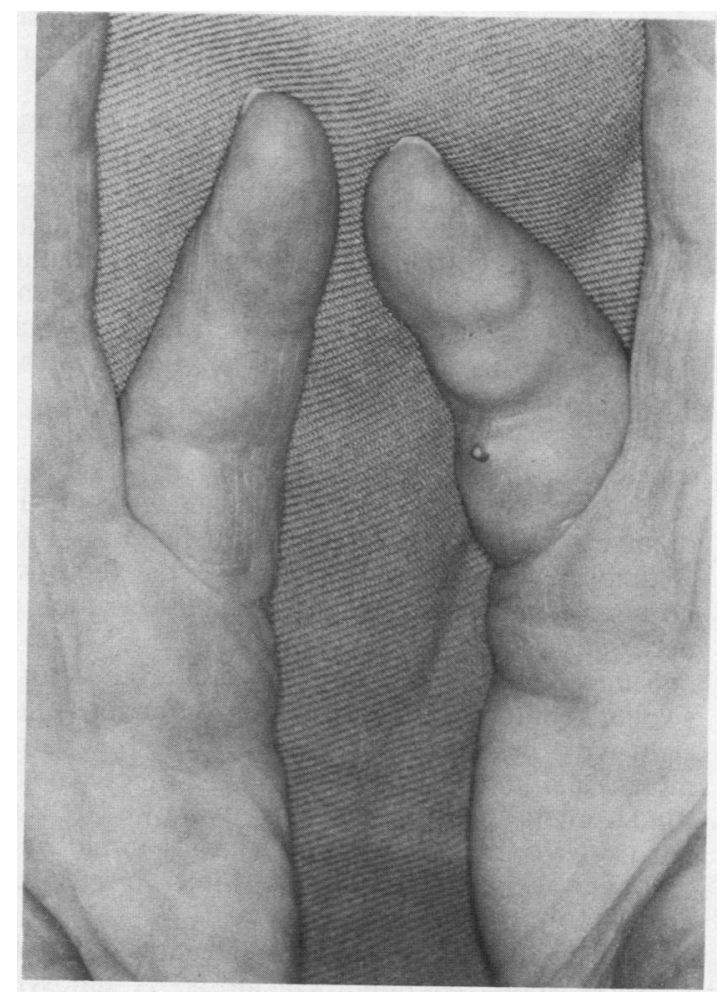

Fig. 1 Case 1. Diffuse swelling of the tendon sheath of the fifth finger. 
changes of the first carpometacarpal joints were also found in the left hand.

Examination of the synovial fluid of the left knee showed $0 \cdot 2 \times 10^{9}$ leucocytes/l with rare intracellular positive birefringent crystals. Red $S$ alizarin staining was negative. Synovial fluid was removed from the tenosynovial cavity of the finger (25 January 1988); it was gelatinous and contained rare white deposits 2-3 $\mathrm{mm}$ in diameter. $x$ Ray powder diffraction analyses of this material showed CPPD crystals in triclinic form. Tenosynovitis recurred, and a synovectomy was performed in April 1988. Histological examination was performed on slides stained by haematoxylin and eosin and by von Kossa stain for calcium phosphate. It showed a non-specific scar remodelling of the synovium with gelatinous deposits and only an isolated small area of CPPD crystal deposits. Recovery was uneventful.

\section{CASE 2}

In February 1988 a 77 year old man developed nodular thickening of the palmar surface of his left wrist. He was also suffering from pain in the first carpometacarpal joints bilaterally and from chronic pain in the left knee with some acute typical attacks of pseudogout. Examination of the left wrist showed a distended synovial sheath of the flexor carpi ulnaris $0.8 \mathrm{~cm}$ in diameter.

There were no signs of carpal tunnel syndrome. $x$ Ray examination showed chondrocalcinosis of both knees but no chondrocalcinosis of the wrists.

Aspiration of the synovial fluid from the tenosynovial cavity produced a clear fluid containing $1.3 \times 10^{9}$ leucocytes $/ 1$ ( $98 \%$ mononuclear) with plenty of intracellular positive rod-like birefringent crystals. A topical corticosteroid agent was injected with a good result.

\section{CASE 3}

A 53 year old woman had suffered from tenosynovitis of the extensor tendon sheaths of the fingers in 1965. A tenosynovectomy was performed, but there was no histological examination. In 1986 the symptoms occurred again.

Clinical examination was normal except for a swollen tendon sheath of the extensor tendons of the right wrist and for a tenderness of the first carpometacarpal joint. Examination of $0.5 \mathrm{ml}$ of synovial fluid from the tendon synovial cavity showed $0.9 \times 10^{9}$ leucocytes/1 (98\% mononuclear) with intracellular and extracellular positive rod-like birefringent crystals.

An $x$ ray examination of the hand and knees failed to show signs of chondrocalcinosis. A tenosynovectomy was performed in November 1986. The synovial fluid contained crystals. Histological examinatiuon was performed on stained (haematen. oxylin and eosin and von Kossa stain) and unstaine slides, which were examined by polarised light. If? showed a non-specific synovial hyperplasia with scae? remodelling but without CPPD crystal deposits흔 Examination of some fragments by transmitteff electron microscopy and by $x$ ray electron diffraction did not show CPPD crystals.

\section{Discussion}

The common feature of these three cases is a hanct tenosynovitis whose exudated synovial fluid con 0 tained CPPD crystals which were disclosed by $x$ ray diffraction in one case and by the presence of rod ${ }^{\infty}$ like positive birefringent crystals in the others. Inpo only one case were CPPD crystal deposits found in the neighbouring synovium and this patien? had presented with radiological signs of chondro $\gg$ calcinosis in both knee and wrist joints. In the other two cases chondrocalcinosis was shown by $x$ raye examination in only one of them.

These cases show that CPPD crystal deposits iro the hand in extra-articular locations induce not only a carpal tunnel syndrome but also tenosynovitis even in the absence of radiological signs of chondro calcinosis elsewhere in the joints. Synovial fluid examination seems to be more simple and sensitive than a synovial biopsy, particularly as histologica $\mathbb{B}$ changes are not equally spread. It is uncertain whether the primary CPPD crystal deposits involve the tenosynovial membrane or the tendon itself.

The authors thank Professor C A Baud, Institute of Morphology University Hospital, Geneva, for $x$ ray diffractions and $\operatorname{Dr} \bar{C}$ Simonetta, Permanence Chirurgicale de Longeraie, Lausanne, fớ clinical data.

\section{References}

1 Bywaters E G L, Hamilton E B D, Williams R. The spine in idiopathic haemochromatosis. Ann Rheum Dis 1971; 30: 453-65

2 Ellman M H, Vazquez T, Ferguson L, Mandel N. Calcium $\gg$ pyrophosphate deposition in ligamentum flavum. Arthritio. Rheum 1978; 21: 611-3.

3 Lagier R. L'approche anatomo-pathologique du concept dêల chondrocalcinose articulaire. Rhumatologie 1981; 33: 421-37.

4 Nagashima C, Takahama M, Shibata T, et al. Calciuno pyrophosphate dihydrate deposits in the cervical ligament flava causing myeloradiculopathy. J Neurosurg 1984; 60: 69-80

5 Gerster J C, Lagier R, Boivin G. Olecranon bursitis related to calcium pyrophosphate dihydrate crystal deposition disease Clinical and pathologic study. Arthritis Rheum 1982; 25: 989-96ळ

6 Gerster J C, Baud C A, Lagier R, Boussina I, Fallet G H? Tendon calcifications in chondrocalcinosis. Arthritis Rheum 1977; 20: 717-22.

7 Gerster J C, Lagier R, Boivin G. Achilles tendinitis associate with chondrocalcinosis. J Rheumatol 1980; 7: 82-9.

8 Leisen J C, Austad E D, Bluhm G B, Sigler J W. The tophus i⿱艹 calcium pyrophosphate deposition disease. JAMA 1980; 244; 1711-2. 
9 Rothschild B M, Round M J. Subcutaneous crystal deposition in pseudogout. JAMA 1980; 244: 2079-80.

10 Schumacher H R Jr, Bonner H, Thompson J J, Kester W L, Benner J J. Tumor-like soft tissue swelling of the distal phalanx due to calcium pyrophosphate dihydrate crystal deposition. Arthritis Rheum 1984; 27: 1428-32.

11 Grahame R, Sutor D J, Mitchener M B. Crystal deposition in hyperparathyroidism. Ann Rheum Dis 1971; 30: 597-604.

12 Dirheimer Y, Bensimon C, Christmann D, Wackenheim C. Syndesmo-odontoid joint and calcium pyrophosphate dihydrate deposition disease. Neuroradiology 1983; 25: 319-21.

13 Bywaters E G L, Dixon A St J, Scott J. Joint lesion of hyperparathyroidism. Ann Rheum Dis 1963; 22: 171-87.

14 Weinstein J D, Dick H M, Grantham S A. Pseudogout, hyperparathyroidism, and carpal tunnel syndrome: a case report. J Bone Joint Surg [Am], 1968; 50: 1669-74.
15 Rubinstein H M, Shah D M. Pseudogout. Semin Arthritis Rheum 1973; 2: 259-80.

16 Spiegel P G, Ginsberg M, Skosey J L, Kwong P. Acute carpal tunnel syndrome secondary to pseudogout: case report. Clin Orthop 1976; 120: 185-7.

17 Goodwin D R A, Arbel R. Pseudogout of the wrist presenting as acute median nerve compression. J Hand Surg [Br] 1985; 10: 261-2.

18 Gerster J C, Lagier R, Boivin G, Schneider C. Carpal tunnel syndrome in chondrocalcinosis of the wrist. Clinical and histologic study. Arthritis Rheum 1980; 23: 924-31.

19 Lagier R, Boivin G, Gerster J C. Carpal tunnel syndrome associated with mixed calcium pyrophosphate dihydrate and apatite crystal deposition in tendon synovial sheath. Arthritis Rheum 1984; 27: 1190-5. 